\title{
Tension Pneumothorax After Emergency Intubation: a Case Report and Review of the Literature
}

\section{Zhong-hua Zhang}

Nanjing Jinling Hospital: East Region Military Command General Hospital, Nanjing Jinling Hospital:

East Region Military Command General Hospital

\section{Zhi-yang Yu}

Nanjing Jinling Hospital: East Region Military Command General Hospital, Nanjing Jinling Hospital:

East Region Military Command General Hospital

\section{Yang Liu}

Nanjing Jinling Hospital: East Region Military Command General Hospital

Cong Liu ( 1033290210@qq.com )

Jinling Hospital, Nanjing University School of Medicine

\section{Case report}

Keywords: emergence, intubation, tension pneumothorax

Posted Date: September 3rd, 2021

DOl: https://doi.org/10.21203/rs.3.rs-847309/v1

License: (c) (i) This work is licensed under a Creative Commons Attribution 4.0 International License.

Read Full License 


\section{Abstract}

Introduction:

Tension pneumothorax during the emergency airway is a rare but deleterious event, which may cause severe cardiorespiratory collapse, leading to brain damage or even death.

\section{Case presentation:}

A 34-year-old male patient was admitted with sudden chest pain. He was diagnosed with acute myocardial infarction and his chest X-ray did not show pneumothorax. The patient after intubation presents emergent complications and was gave treatment.

\section{Discussion and Conclusions:}

Tension pneumothorax in tracheal intubation of emergency is a more rare but deleterious event, especially when predisposing factors cannot be known in view of acute profound hypoxemia. We collect several rare cases of tension pneumothorax of different etiology and drawing lessons from the past.

\section{Introduction}

The emergency airway with the 'cannot ventilate' situation contributes to a high percentage of anesthesiarelated morbidity and mortality. Tension pneumothorax during the emergency airway is a rare but deleterious event, which may cause severe cardiorespiratory collapse, leading to brain damage or even death. Under these conditions, the development of tension pneumothorax is a well-established phenomenon, necessitating fast and proper diagnosis and rapid drainage(1). This case is an example of acute myocardial infarction with tachycardia and respiratory distress, necessitating intubation and ventilation, comorbid with tension pneumothorax. This life-threatening situation can be preventable with the appropriate awareness. There is no consensus on management, which is only guided by the experience of case series. Families of the patient gave informed consent for publication.

\section{Case Presentation}

A 34-year-old male patient was admitted with sudden chest pain. In transit, the patient suddenly developed ventricular fibrillation. And the cardiopulmonary resuscitation was performed. After admission, rapid management was done including blood test, electrocardiograph and chest $\mathrm{X}$-ray. He was diagnosed with acute myocardial infarction and his chest X-ray did not show pneumothorax. In order to maintain vital signs, he had an operation of Intra-Aortic Balloon Pump in the Emergence room. Eight hours later, the patient was observed to be in severe respiratory distress with tachypnoea and nasal flaring. Tympanic temperature was $36.8^{\circ} \mathrm{C}$, peripheral pulse rate 100 beats $/ \mathrm{min}$, respiratory rate 40 breaths $/ \mathrm{min}$, and blood pressure $97 / 70 \mathrm{mmHg}$. Arterial blood gases on a 3-L oxygen mask were as follows: $\mathrm{PH} 7.18, \mathrm{PaO}_{2}$ $40 \mathrm{mmHg}, \mathrm{PaCO}_{2} 72.0 \mathrm{mmHg}, \mathrm{HCO}_{3} 26 \mathrm{mmol} / \mathrm{L}$, lactic acid $10.1 \mathrm{mmol} / \mathrm{L}$ and arterial oxygen saturation 
$64 \%$. On these medications (cardiac tonic, and vasoactive drugs), the patient was still distressed without any improvement in his condition. Considering the acute heart failure, the anesthetist was invited for further diagnosis and treatment. For improving oxygen balance, the anesthetist advised tracheal intubation. An video- guided intubation was performed with a $7.0 \mathrm{~mm}$ tube and a video laryngoscope. Placement should be confirmed with bilateral breath sounds, symmetric chest rise, and capnography, however, the auscultation breath sound was not clear and chest percussion revealed hyperresonance bilaterally. The patient's oxygen saturation declined from $98-54 \%$, and his heart rate decreased from 100 to $39 \mathrm{bpm}$. At the same time, blood pressure cannot be measured. Therefore, the cardiopulmonary resuscitation was initiated. Approximately 10 minutes following confirmation of endotracheal tube placement, the patient required higher positive pressures during manual ventilation. On connecting the endotracheal tube to the ventilator, volume controlled and synchronized intermittent mandatory ventilation mode with an inspiratory oxygen fraction:0.7, tidal volume: $450 \mathrm{ml}$, respiratory rate: 16 beat/min. Peak inspiratory pressure ranged between 45 and $50 \mathrm{cmH}_{2} \mathrm{O}$. As a young resident doctor, incorrect intubation would be the first consideration. The patient was extubated and reintubated with video laryngoscopy again, but a copious quantity of blood obscured the video picture, without any improvement in the patient's condition. Senior anesthetist was called to help hurriedly. A suction catheter was placed easily through the tube and produced only a scant amount of blood and secretions. Finding ventilator monitor tidal volume of $34 \mathrm{ml}$, changing ventilation mode was been tried, but there was no difference. On reflection, he found the presence of severe respiratory distress, a hyperresonance, air entry diminished and the presence of no blood pressure. He alerted the attending clinicians to entertain other possible diagnoses including the obstruction of the endotracheal tube, tension pneumothorax, esophageal intubation, severe bronchospasm, or mainstem bronchus intubation. Placement of tube was confirmed by the video laryngoscopy obscurely. He thought tension pneumothorax should be the first possible consideration. Therefore, he placed the syringe needle in the 2 nd intercostal space at midclavicular line with immediate drainage of bubbles. Management was therefore directed at the immediate relief of symptoms without waiting for radiographic confirmation of the diagnosis. Surprisingly, heart rate and blood pressure, airway pressure got normal gradually. Due to the patient's multiple comorbidities and poor prognosis, the patient's families held a goals of care discussion with doctors. Finally, they decided to give up artificial life support including thoracostomy tube.

\section{Discussion And Conclusions}

The incidence of occult pneumothorax is reported to be $5 \%$ in literature(2). Tension pneumothorax in tracheal intubation of emergency is a more rare but deleterious event, especially when predisposing factors cannot be known in view of acute profound hypoxemia. Tension pneumothorax both are negatively impacted by positive ventilation and has similar hemodynamic and respiratory consequences, while at the same time, extreme air-trapping can also lead to barotrauma(3). We collect several rare cases of tension pneumothorax of different etiology. Sunil Kumar Garg(4) and Erik Nygaard Andresen(5) present iatrogenic pneumothorax. latrogenic pneumothorax is a complication caused by procedures such as transthoracic needle aspiration, thoracentesis, transbronchial biopsy, pleural biopsy, and positive 
pressure ventilation. Even, nebulization and nasogastric feeding tube can cause similar phenomenon because of misuse. Therefore, education and training should be a continuous process. And approaches to all interventions should be taught to all medical workers so that they can be in a better position to understand the consequences of any deviation from standard. Yuki Yoshimatsu(6) and Khoa Anh Nguyen(7) describe the same situation because of the patient's own special condition including neurofibromatosis -related lung disease and congenital tracheal stenosis. High-resolution chest CT of patients, of course, may show anatomical abnormality including bullae, cysts, emphysema, and ground glass opacities. And congenital abnormality like tracheal stenosis and emphysematous could exist in absence of clinical symptoms and signs. Needless to say, knowing the patient's laboratory tests and examinations in detail are very essential. Ayman(8) and Steven(9) think present history of patients is also important, which include asthma, drug addiction and so on. Asthma-related airway inflammation and alpha-1 antitrypsin deficiency caused by tobacco use and cocaine were the major cause for emphysematous, which is inducing factor of pneumothoraxes and the rupture of bullae. The rupture of bullae during manual ventilation is more serious by the rapid cardiorespiratory deterioration after intubation and start of controlled ventilation(1). If signs of circulatory compromise are not obvious and other indirect signs like jugular vein enlargement remain unnoticed, the diagnosis could be set with great delay. And the patient mentioned above had a known history of smoking and drinking, which may be the cause of pneumothoraxes.

The inability to ventilate a patient after successful intubation is a rare but emergent situation and may be caused by obstruction of the endotracheal tube, mainstem bronchus intubation, esophageal intubation, severe bronchospasm, or bilateral tension pneumothorax(10). It is recommended that observation of small occult pneumothoraxes, without tube thoracostomy in patients not receiving mechanical ventilation, is safe(8). But, in patients undergoing mechanical ventilation, chest tube is indispensable appliance to prevent development of tension pneumothorax. As for confirmation of tracheal tube placement, observation of passing through vocal cords during laryngoscopy, auscultation, observing symmetrical chest expansion and observation capnography or end-tibal carbon dioxide can be valid(11). However, the fiberoptic scope was not advised, on account of poor quality of the image obscured by secretions and blood, especially in blunt trauma patients.

Drawing lessons from the past, firstly, the patient should be fully evaluated before intubation including laboratory tests and examinations, present history and physical examination like auscultation of breath sounds in both lungs and palpation of the abdomen. Secondly, early control of the airway to ensure oxygenation, ventilation and protection against aspiration is paramount. Capnography to measure endtibal carbon dioxide is a very practical prop, especially when auscultation breath sound is not clear. When intubation complications occur, the cannula should not be hastily removed and the patient's symptoms should be carefully evaluated again. In the emergency intubation items, if pulmonary shunt is present from the collapsed or atelectatic lung, the low mixed venous $\mathrm{PO}_{2}$ from low cardiac output will cause arterial hypoxemia. Breath sounds must be assessed immediately after tube insertion and periodically thereafter. Thirdly, if at any time breath sounds become decreased or lost, the position of the 
endotracheal tube has been confirmed before assuming that the patient has suffered a hemothorax or pneumothorax. Patients exhibited both hypoxemia, hypotension and signs of low cardiac output. They probably should elevate jugular venous pressure from impeding of venous return, which would cause an elevated intracranial pressure. The resultant increased intrathoracic pressure impeded venous blood return and ultimately led to cardiovascular collapse. Therefore, the diagnosis of tension pneumothorax can be proved by the patient's clinical signs listed as before. Tension pneumothorax was suspected and should be managed with insertion of an 18-gauge intravenous catheter in the second intercostal space, followed by chest tube insertion.

\section{Abbreviations}

PH Pondus Hydrogenii

$\mathrm{PaO}_{2}$ Partial Pressure of Oxygen

$\mathrm{PaCO}_{2}$ Partial Pressure of Carbon Dioxide

CT Computed tomography

\section{Declarations}

The authors declare that they have no competing interests. Ethics had been approved.

\section{Consent for publication}

Written informed consent was obtained from the patient's next of kin or family for publication of this Case report and any accompanying images. A copy of the written consent is available for review by the Editor of this journal

\section{Authors' contributions}

Authors including $Z H Z, Z Y Y, Y L$, participated in the care of the patient. LC helped draft and revise this manuscript, and read and approved the final manuscript.

\section{Acknowledgement}

Not applicable

\section{Funding}

Not applicable.

\section{Availability of data and materials}

Not applicable. 


\section{Ethics approval and consent to participate}

Not applicable.

\section{References}

1. Amaniti E, Provitsaki C, Papakonstantinou P, Tagarakis G, Sapalidis K, Dalakakis I, et al. Unexpected Tension Pneumothorax-Hemothorax during Induction of General Anaesthesia[J].Case reports in anesthesiology. 2019,2019:1-4.

2. Omar HR, Abdelmalak H, Mangar D, Rashad R, Helal E, Camporesi EM. Occult pneumothorax, revisited[J].J Trauma Manag Outcomes. 2010,4:12.

3. Whitaker DK, O'Sullivan EP. Capnography, Esophageal Intubation, and Capnomanaging Cardiac Arrests in the Operating Room[J].Anesth Analg. 2018,127(3):e48-e9.

4. Garg SK, Garg P, Anchan N, Jaiswal A. latrogenic Bilateral Simultaneous Pneumothorax: Call for Vigilance[J].Indian journal of critical care medicine : peer-reviewed, official publication of Indian Society of Critical Care Medicine. 2017,21(9):607-9.

5. Andresen EN, Frydland M, Usinger L. Deadly pressure pneumothorax after withdrawal of misplaced feeding tube: a case report[J].Journal of medical case reports. 2016,10:30.

6. Yoshimatsu Y, Morita R, Suginaka M, Furukawa K, Nakamura N, Yamairi K, et al. Difficult intubation due to unknown congenital tracheal stenosis in the adult: a case report and literature review[J].Journal of thoracic disease. 2018,10(2):E93-E7.

7. Nguyen, Khoa Anh et al. Neurofibromatosis type 1: a case highlighting pulmonary and other rare clinical manifestations. [J].BMJ case reports vol. Jan. 2018, doi:10.1136/bcr-2017-222614

8. Metry AA. Acute severe asthma complicated with tension pneumothorax and hemopneumothorax. Int J Crit Illn Inj Sci 2019;9:91-5.

9. Douedi S, Upadhyaya VD, Patel I, Mazahir U, Costanzo E, Hossain MA. Cocaine-Induced Giant Bullous Emphysema[J].Case reports in medicine. 2020,2020:6410327.

10. Liberman JS, Weigel W, Neal JM. Difficult Ventilation After Successful Intubation in the Emergency Setting due to a Ball Valve Clot[J].A \& A case reports. 2016,6(10):291-2.

11. Hossein-Nejad H, Payandemehr P, Bashiri SA, Nedai HH. Chest radiography after endotracheal tube placement: is it necessary or not?[J].Am J Emerg Med. 2013,31(8):1181-2. 